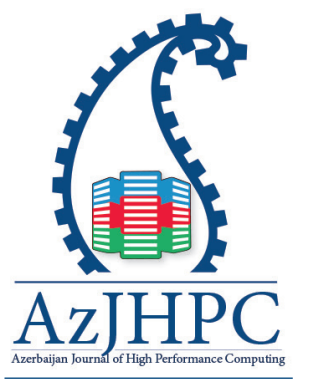

${ }^{*}$ Correspondence: Pragnan Chakravorty, Clique for Applied

Research in Electronic Technology, India, pciitkgp@ieee.org

\title{
Antenna Array Failure Correction Using Improved Particle Swarm Optimization with Wavelet Mutation
}

\author{
Pallavi Mitra1 ${ }^{1}$, Durbadal Mandal ${ }^{1}$, Rajib Kar ${ }^{1}$, Pragnan Chakravorty² \\ ${ }^{1}$ National Institute of Technology Durgapur-713209, India, pallavi08091992@gmail.com, \\ durbadal.bittu@gmail.com \\ ${ }^{2}$ Clique for Applied Research in Electronic Technology, India, pciitkgp@ieee.org
}

\section{Abstract}

The advent of complex engineering systems has made computation intensive design processes inevitable. Unfortunately, such processes require larger resources, i.e., larger memory, parallel processing, etc., and time than usual. As a result, the high performance issues like managing these processes to run within limited resource pools has become ever more challenging. This paper reports an application of intelligent computing in the specific area of antenna arrays and implicitly shows how a computationally intensive process can improve its performance within a limited available resource by judiciously combining different algorithms. Failures in radiating elements in an antenna array usually increase the undesired side lobe radiation, thereby distorting the original radiation pattern. Here, restoration of the original pattern has been attempted by restricting the side lobe levels (SLLS) to the desired threshold using a meta-heuristic optimization technique named Improved Particle Swarm Optimization with Wavelet Mutation (IPSOWM). Also, as a reference, the performance of this algorithm has been compared with Improved Particle Swarm Optimization (IPSO) technique. Results show that IPSOWM yields a better solution to the existing problem as compared to IPSO.

Keyword: Antenna Array, Particle Swarm Optimization, Wavelet Mutation, Side Lobe Level

\section{Introduction}

Antenna arrays are extensively used in numerous wireless communication systems like mobiles, sonar, satellite communication, and so on; its use has seen higher prominence in some latest research areas like 5G communications. The total radiating components in the antenna array can be as small as two or can be as large as a few thousand. Due to the presence of such a huge number of elements, a situation may always arise when one or more than one element fails, either partially or completely. Owing to the failure of elements in the array, sharp variations across the array in field intensity might occur and it distorts the pattern by regulating the sidelobe-level. There is a decline in the performance of the wireless communication 
system's where the antenna array is housed. Therefore, it is necessary to synthesize the optimal beam pattern with a specified SLL to improve the system performance because it might not be practically feasible, every time to replace the failed antenna element. The replacement may not prove to be cost effective and can be timeconsuming though it will always give a correct solution. Secondly, in space platforms, the replacement of the failed antenna array element is not possible. The taper of the antenna array distribution is increased to decrease the side-lobes to the required level. However, the solution has numerous drawbacks. Firstly, because the side-lobe level of the design for the array reduces, there will be an increase in excitation value for the sensitivity of SLL to perturbation. Second, the leading beams design taper can be changed by to an unacceptable level and Finally, absence of the desired symmetry or smooth out sharp field variations cannot be merely compensated by increasing the taper level. The defective components compensation can be found out by arithmetically calculating the excitation value of every non-defective component, which will optimize the function. This function takes the location of the failed elements into account.

Many techniques have been suggested to solve this problem. Jung-Hoon Han gave an algorithm for the re-synthesizing distorted beam of the optimal beam pattern. T.J Peters gave an algorithm which uses the single element failure conditions to retrieve the directional pattern of the linear antenna array(LAA). Wang, L.-L. et al. proposed a combination of FFT for array failure correction and genetic algorithm. Beng-Kiong Yeo proposed an algorithm in digital beamforming of different arrays based on their array failure correction. We have tried to restore the original radiation pattern or obtain the required radiation pattern with a stipulated SLL, by the implementation of IPSOWM technique. Also, as a reference, the algorithm has been compared with the IPSO technique. For testing the algorithm mentioned above, a linear isotropic antenna array is considered. It can also be used for the planar arrays as well. A comparative study of both algorithms has been made. Both Optimization techniques can be compared using the numerical results that we obtain.

\section{Design Equation}

Linear antenna array structure:

The N-element linear array which is in progressive phase excitation has an array factor (AF) which is expressed by the below equation:

$$
A F=\sum_{n=1}^{N} w_{n} e^{j(n-1) d \cos \theta}
$$

Where $\mathrm{N}=$ Total number of array elements.

$\mathrm{w}_{\mathrm{n}}=$ Non-uniform excitation of every element.

$\mathrm{d}=$ Spacing between every element.

$\theta=$ Broadside angle.

The degradation of null regions is due to antenna arrays element failure, and they also lead to pointed variations in the electric field. As a result, both the ripple level and side-lobe of power pattern is increased. So our objective was determining weights of different elements of the array to keep the zenith of the SLL of disrupted array near the original array.

Linear antenna array has been used in this thesis. Here, elements are kept at a 


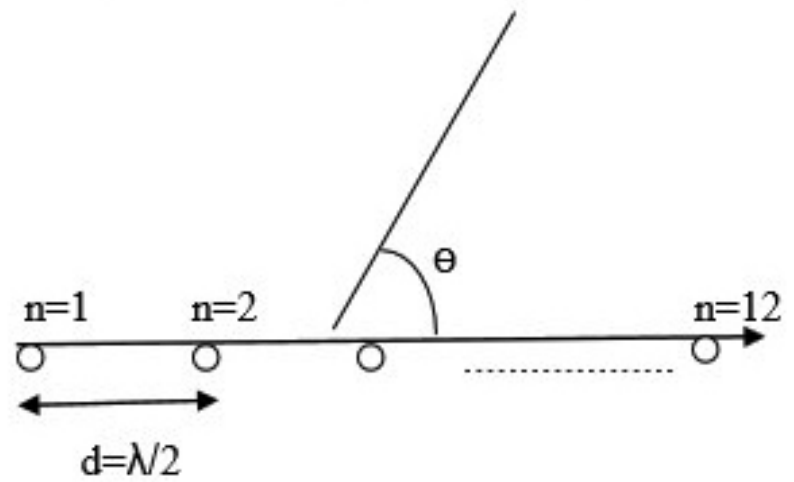

Fig. 1. Symmetric Linear Antenna Array Structure of 12 elements placed along $x$-axis

distance of (lambda/2) from each other on either side of the origin, where lambda is the wavelength. However, this technique could be applied to any array with a complex geometrical shape.

\section{Evolutionary optimization techniques employed:}

A. Particle Swarm Optimization: PSO is stochastic flexible optimization technique, which is population-based. Contrary to the traditional optimization methods, PSO will efficiently handle the complex, non-continuous and non- differential single objective functions. Due to the space limitations of PSO, the details of them are not given. Furthermore, some references on PSO are given in [1-13]. Velocities of different elements are altered in the PSO by the following equation given below,

$$
V_{i}^{(k+1)}=w V_{i}^{k}+C_{1} \text { rand }_{1}\left(\text { pbest }_{i}^{k}-S_{i}^{k}\right)+C_{2} \text { rand }_{2}\left(\text { gbest }^{k}-S_{i}^{k}\right)
$$

The particles searching positions may be altered in solution space by equation(3)

$$
S_{i}^{(k+1)}=S_{i}^{k}+V_{i}^{(k+1)}
$$

B. Improved Particle Swarm Optimization: For enhancing the total searchability of the PSO, a small review is taken into account with the help of the below-mentioned changes. The changed PSO is called as IPSO [13-16].

Finally, the $i^{\text {th }}$ particle's $j^{\text {th }}$ component modified velocity is shown by

$$
\begin{array}{rl}
V_{i}^{(k+1)}=r_{2} & * \operatorname{sign}\left(r_{3}\right) * V_{i}^{k}+\left(1-r_{2}\right) \cdot C_{1} * r_{1} *\left\{\text { pbest } t_{i}^{k}-S_{i}^{k}\right\}+ \\
& +\left(1-r_{2}\right) * C_{2} *\left(1-r_{1}\right) *\left\{\text { gbest }^{k}-S_{i}^{k}\right\}
\end{array}
$$

Here the random numbers between 0-1 are denoted by $r_{1}, r_{2}$ and $r_{3}$ and the function $\operatorname{sign}\left(r_{3}\right)$ is described

$$
\operatorname{sign}\left(r_{3}\right)=-1 \text { when } r_{3}<0.05,=1 \text { when } r_{3}>0.05
$$

Then, In the solution space, searching points of the particles can be altered by the equation (3).

\section{IPSO with the wavelet mutation (IPSOWM)}

Basic wavelet theory: Concept

Wavelet with a limited duration can be used in different seismic signals and they could be changed by mixing the translations with dilations of oscillatory function. 
Wavelet transforms categorized into 2, namely: continuous and discrete wavelet transform. Continuous wavelet transform $W_{a, b}(x)$ of the function $\mathrm{f}(\mathrm{x})$ w.r.t a mother wavelet

$\psi(\mathrm{x}) \in \mathrm{L}^{2}(\mathfrak{R})$ is given by the equation [17-20].

$$
\begin{gathered}
W_{a, b}(x)=\frac{1}{\sqrt{c_{\psi}}} \int_{-\infty}^{+\infty} f(x) \psi_{a, b}^{*}(x) d x \\
\psi_{a, b}(x)=\frac{1}{\sqrt{a}} \psi\left(\frac{x-b}{a}\right) ; x \in \Re, a, b \in \Re, a>0
\end{gathered}
$$

In (6), dilation (scale) parameter is denoted by $a$ and translation (shift) parameter is denoted by $b .\left(^{*}\right)$ denotes the complex conjugate. It can be seen that the spread of the wavelet is controlled by a and control position is regulated be $b$. The mother wavelet From scaling and shifting a set of basis function is derived $\psi_{\mathrm{a}, \mathrm{b}}(\mathrm{x})$. The daughter wavelet is the basis function of the transform. The mother wavelet should obey this condition given below:

$$
C_{\psi}=2 \pi \int_{-\infty}^{+\infty} \frac{|\bar{\psi}(\omega)|^{2}}{\omega} d \omega<\infty
$$

where $\bar{\psi}(\omega)$ is the Fourier transform of $\psi(\omega)$ and given by the following equation.

$$
\bar{\psi}(\omega)=\frac{1}{\sqrt{2 \pi}} \int_{-\infty}^{+\infty} \psi(x) e^{-j \omega x} d x
$$

Majority of the energy of $\psi(x)$ is restricted to a bounded finite domain.

The partnership of Wavelet-based Mutation with IPSO (IPSOWM)

Every component of the particles of the limited population will mutate and the randomly selected $j^{\text {th }}$ element and an $\mathrm{i}^{\text {th }}$ particle at $k^{\text {th }}$ iteration $\left(\mathrm{s}_{\mathrm{i}, \mathrm{j}}^{(\mathrm{k})}\right)$ will undergo mutation represented by the following equation given below:

$$
s_{i, j}^{(k)}= \begin{cases}S_{i, j}^{(k)}+\sigma\left(S_{j}, \max -S_{i, j}^{(k)}\right) & \text { if } \sigma>0 \\ S_{i, j}^{(k)}+\sigma\left(S_{i, j}^{(k)}-S_{j, \min }\right) & \text { if } \sigma \leq 0\end{cases}
$$

where $\sigma=\psi_{\mathrm{a}, 0}(\mathrm{x})=\frac{1}{\sqrt{\mathrm{a}}} \psi\left(\frac{\mathrm{x}}{\mathrm{a}}\right)$

A Morlet wavelet which is shown in Fig. 2. (mother wavelet) is defined in the following equation.

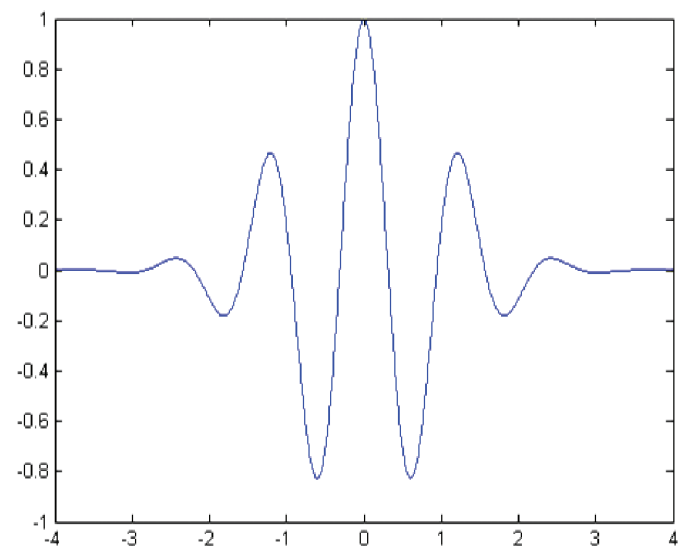

Fig. 2. Morlet wavelet. 


$$
\begin{gathered}
\psi(x)=e^{\frac{-x^{2}}{2}} \cdot \cos (5 x) \\
\text { Thus, } \sigma=\frac{1}{\sqrt{a}} e^{\frac{-\left(\frac{x}{a}\right)^{2}}{2}} \cos \left(5\left(\frac{x}{a}\right)\right)
\end{gathered}
$$

Fig. 3 shows the various dilated Morlet Wavelets. From this figure amplitude of $\psi_{\mathrm{a}, 0}(\mathrm{x})$ is scaled down as $a$ increases. This theory will be utilized in mutation operations to increase the performance of searching.
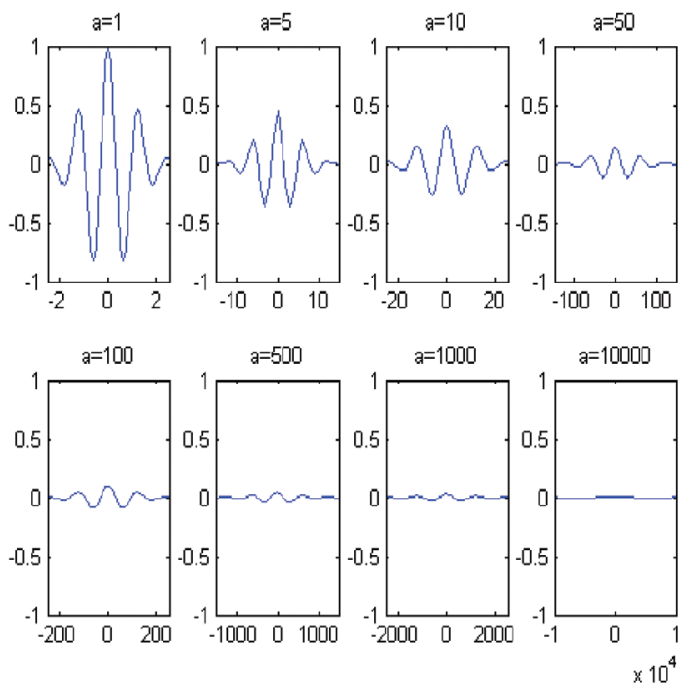

Fig. 3. Morlet wavelet dilated by different values of parameter a.

More than $99 \%$ of total energy is situated in the interval $[-2.5,2.5]$ and $x$ could be generated randomly in the interval [-2.5 $\mathrm{x}$ a, $2.5 \times \mathrm{x}$ ]. To meet the fine-tuning purpose, the value of the dilation parameter $a$ is varied with the $k / K$ value, and $K$ denotes the maximum number of iteration cycles, $k$ denotes current iteration cycle. Therefore, $a$ monotonic increasing function with $a$ and $k / K$ is written by the following equation [1314]

$$
a=e^{-\ln (g)\left(1-\frac{k}{K}\right)^{\xi \omega m} \omega m+\ln (g)}
$$

where $g$ is an upper limit of the parameters is a monotonic increasing function's shape parameter, the iteration cycle $\mathrm{k}$ varies from 1 to a maximum number of iteration cycles, $\mathrm{K}$ which is the value of $a$ varies from 1 to $\mathrm{g}$. The perfect balance that substantially affects the execution of the IPSOWM is regulated by an ideal choice of control parameters, which are the probability of mutation $\left(p_{m}\right)$, the shape parameter of WM $\xi_{\omega \mathrm{m}}$, swarm size $\left(n_{p}\right)$, and $\xi_{\omega \mathrm{m}}$. Modifying the parameter's would change the characteristics of WM. However, the value required to perform fine tuning is regulated by $a$ which in-tum increases the value of $\xi_{\omega m}$. Thus for increasing step size $(\sigma)$ for early mutation, a big value of the $\xi_{\omega m}$ can be used.

To obtain individual ideal values of $g$ and $\xi_{\omega m}$ as 10.0 and 2 , respectively. A continuous sensitivity analysis w.r.t the reliability of $a$ on $(K / K)$ is carried out. 


\section{Results and Discussion}

For both the algorithms, viz IPSOWM and IPSO, the same cost function is used to avoid ambiguity. The initial side-lobe level is set at $-15 \mathrm{~dB}$ and the linear array consists of 8 elements, and one element of the array is presumed to fail. FNBW (Beam Width between First Null) is 28 degrees, with nulls located respectively at 76 degrees(theta1) and 104 degrees(theta2). Initially, the array factor is calculated from 0 degrees to the first null for all the 8 elements as,

$$
A F \text { theta } 1=A F \text { theta } 1+I(m) * \exp (j *(m-1) * \text { pi. } * \text { cosd }(\text { thetal }))
$$

Next, the array factor is again calculated from the second null to the end, for all the 8 elements as,

$$
A F \_ \text {theta } 2=A F \text { theta } 2+I(m) * \exp \left(j *(m-1) * p i{ }^{*} \cos d(\text { theta } 2)\right)
$$

The zenith of the main lobe is located at 90 degrees(theta3), and again the array factor is calculated as,

$$
A F_{-} \text {theta }_{3}=A F_{-} \text {theta } 3+I(m) * \exp (j *(m-1) * p i . * \cos d(\text { theta } 3))
$$

Next, the cost function is designed as,

$$
y=\max \left(\left[a b s\left(A F \_t h e t a_{1}\right), a b s\left(A F_{-} \text {theta }\right)\right] / a b s\left(A F_{-} \text {theta }_{3}\right)\right)
$$

Now this cost function, which is to be optimized, is fed to the IPSOWM Algorithm. The total number of iterations is 400 .

Both techniques, namely IPSOWM and IPSO, have been used in the problem of re-synthesis of antenna array radiation patterns. Different Results have been obtained in the form of plots between current excitation and radiation pattern, as shown in Tables 1 and 2.

\section{Table 1: Current Excitation values for IPSO}

\begin{tabular}{|c|c|c|c|c|c|c|c|c|}
\hline $\begin{array}{c}\text { Error } \\
\text { Position }\end{array}$ & \multicolumn{7}{|c|}{ Current Excitation values } \\
\hline 1 & 0 & 0.6243 & 0.6498 & 0.7196 & 0.8380 & 0.6618 & 0.6117 & 0.7742 \\
\hline 2 & 0.3759 & 0 & 0.4775 & 0.6300 & 0.4877 & 0.4819 & 0.4400 & 0.3910 \\
\hline 3 & 0.4809 & 0.6057 & 0 & 0.9267 & 0.7500 & 0.9271 & 0.7237 & 0.3495 \\
\hline 4 & 0.2977 & 0.4752 & 0.6172 & 0 & 0.7659 & 0.5646 & 0.4401 & 0.3948 \\
\hline 5 & 0.5183 & 0.4359 & 0.7796 & 0.9948 & 0 & 0.5976 & 0.4627 & 0.3743 \\
\hline 6 & 0.4152 & 0.5183 & 0.9447 & 0.9277 & 0.9156 & 0 & 0.8147 & 0.5104 \\
\hline 7 & 0.4480 & 0.8004 & 0.9521 & 0.7034 & 0.9323 & 0.7491 & 0 & 0.7415 \\
\hline 8 & 0.9367 & 0.6022 & 0.8863 & 0.9146 & 0.8430 & 0.9880 & 0.7126 & 0 \\
\hline
\end{tabular}

Thus, from table 3, we can conclude that IPSOWM shows good results in reducing SLL than IPSO when there is a failure of one element of the antenna.Also, in various applications, IPSO has a huge iteration time when compared to IPSOWM.

\section{Conclusion}

Both the algorithms have been implemented for the fault correction of a linearantenna array when one element is faulty. On the basis of the above radiation pattern plots and tables showing current excitation values and the side lobe level values(in $\mathrm{dB}$ ), we can conclude that IPSOWM shows good results in reducing SLL than IPSO 
Pallavi Mitra, Durbadal Mandal, Rajib Kar and Pragnan Chakravorty

Table 2: Current Excitation values for IPSOWM

\begin{tabular}{|c|c|c|c|c|c|c|c|c|}
\hline $\begin{array}{c}\text { Error } \\
\text { Position }\end{array}$ & \multicolumn{7}{|c|}{ Current Excitation values } \\
\hline 1 & 0 & 0.9185 & 0.8481 & 0.9200 & 0.9151 & 0.9245 & 0.6400 & 0.8534 \\
\hline 2 & 0.3759 & 0 & 0.4771 & 0.6325 & 0.4873 & 0.4811 & 0.4412 & 0.3910 \\
\hline 3 & 0.4313 & 0.6193 & 0 & 0.7961 & 0.5943 & 0.7516 & 0.5779 & 0.3133 \\
\hline 4 & 0.1717 & 0.5798 & 0.7436 & 0 & 0.8671 & 0.6316 & 0.5564 & 0.4086 \\
\hline 5 & 0.2482 & 0.3002 & 0.5717 & 0.8991 & 0 & 0.5987 & 0.5073 & 0.3752 \\
\hline 6 & 0.4152 & 0.5183 & 0.9447 & 0.9277 & 0.9156 & 0 & 0.8147 & 0.5104 \\
\hline 7 & 0.480 & 0.8004 & 0.9521 & 0.7034 & 0.9323 & 0.7491 & 0 & 0.7415 \\
\hline 8 & 0.8153 & 0.4284 & 0.7084 & 0.6413 & 0.5844 & 0.6208 & 0.4301 & 0 \\
\hline
\end{tabular}

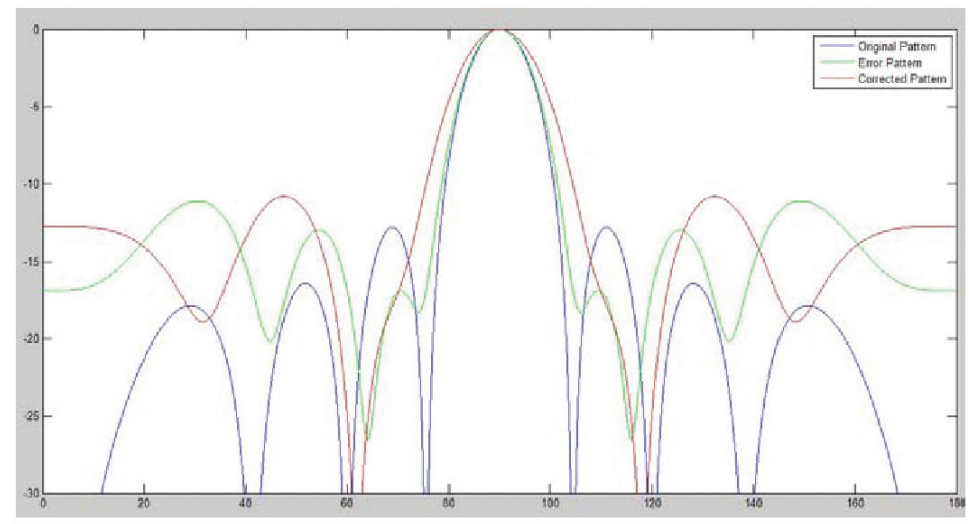

Fig. 4: Radiation pattern using IPSO when the second element is faulty ( $x$-axis (angle in degrees ${ }^{\circ}$ ), $y$-axis: magnitude in $d B$ )

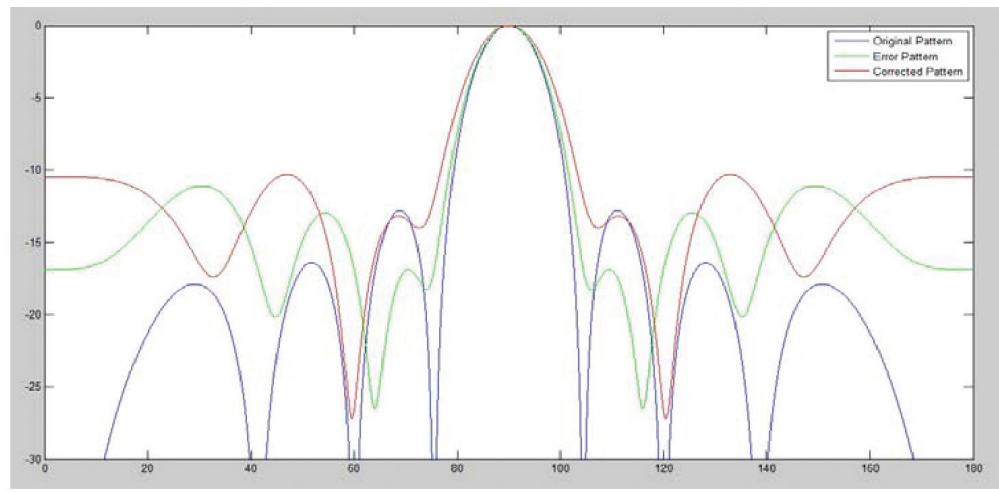

Fig. 5: Radiation pattern using IPSOWM when the second element is faulty( $x$-axis (angle in degrees ${ }^{\circ}$ ), $y$-axis: magnitude in $d B$ ) 


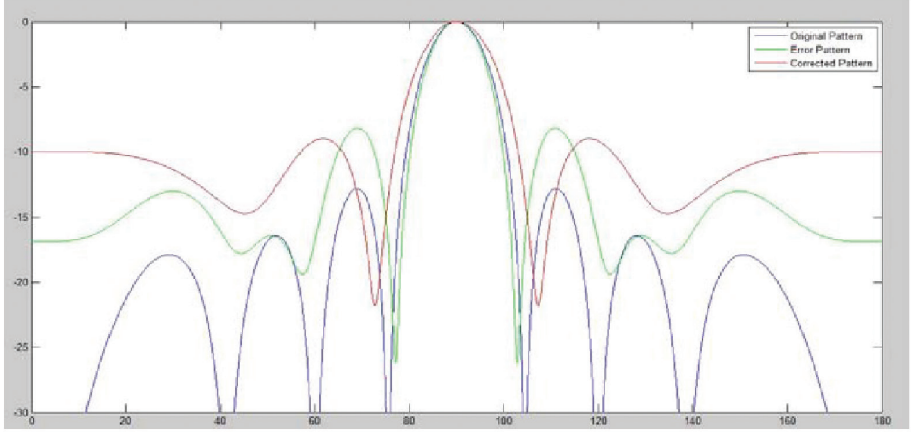

Fig. 6: Radiation pattern using IPSOWM when the fourth element is faulty( $x$-axis (angle in degrees ${ }^{\circ}$ ), $y$-axis: magnitude in $d B$ )

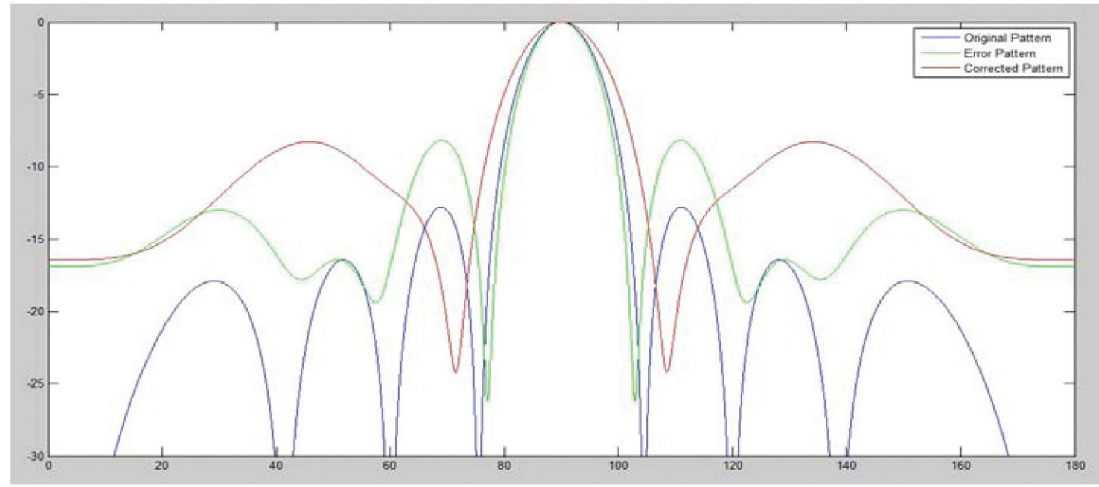

Fig. 7: Radiation pattern using IPSO when the fourth element is faulty(x-axis (angle in degrees $\left.{ }^{\circ}\right), y$-axis: magnitude in $d B$ )

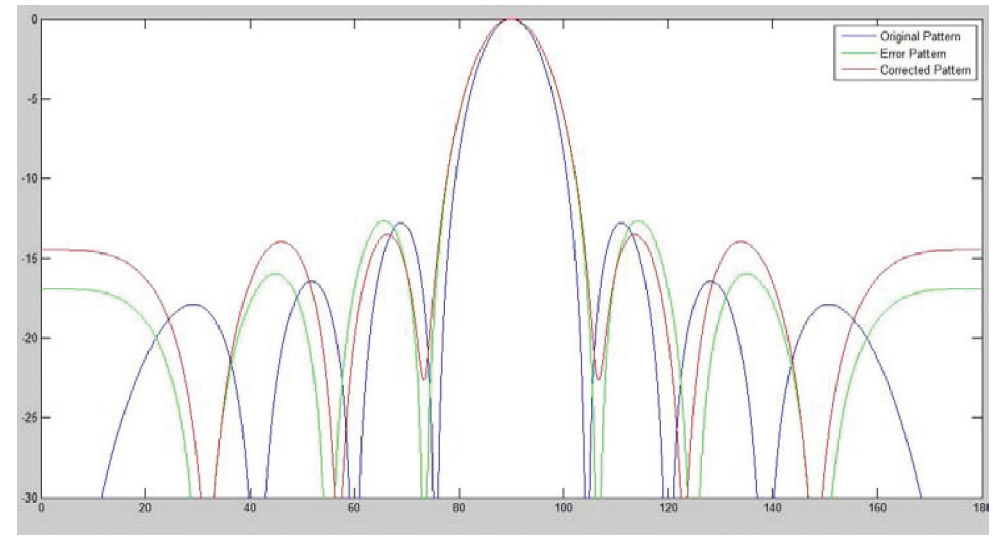

Fig. 8: Radiation pattern using IPSO when the eighth element is faulty ( $x$-axis (angle in degrees ${ }^{\circ}$, $y$-axis: magnitude in $d B$ ) 


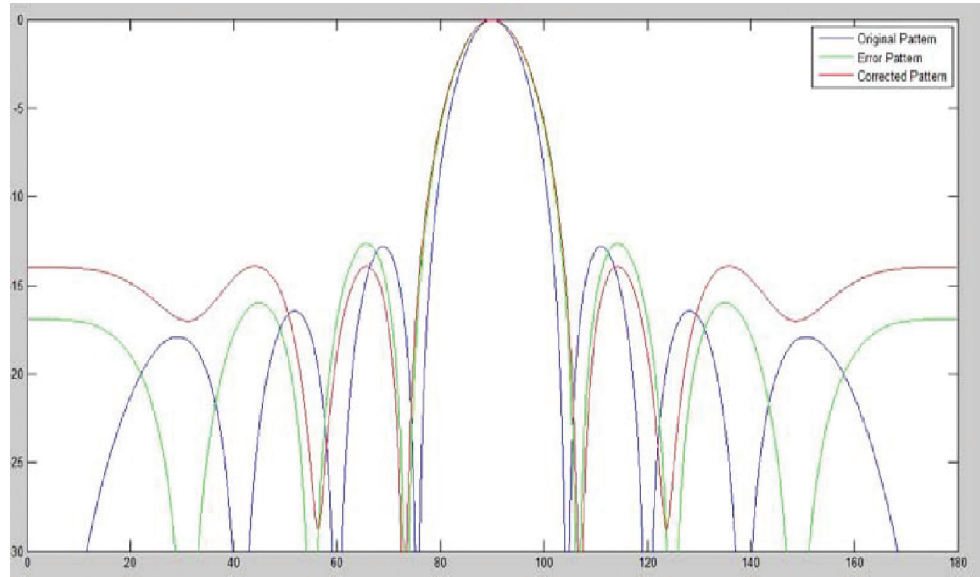

Fig. 9: Radiation pattern using IPSOWM when the eighth element is faulty(x-axis: angle in degrees ${ }^{\circ}, y$-axis: magnitude in $d B$ )

Table 3: Side-lobe level table

\begin{tabular}{|c|c|c|c|c|}
\hline $\begin{array}{c}\text { Fault } \\
\text { Position }\end{array}$ & Original SLL $(\mathrm{dB})$ & Faulty SLL $(\mathrm{dB})$ & $\begin{array}{c}\text { Corrected us- } \\
\text { ing IPSO }(\mathrm{dB})\end{array}$ & $\begin{array}{c}\text { Corrected using } \\
\text { IPSOWM }(\mathrm{dB})\end{array}$ \\
\hline $\mathrm{i}$ & -12 & -11.5 & -14 & -15 \\
\hline 2 & -17.5 & -12.5 & -20 & -25 \\
\hline 3 & -17.5 & -10 & -18.5 & -22 \\
\hline 4 & -12.5 & -9 & -22 & -25 \\
\hline 5 & -14 & -9 & -17 & -24 \\
\hline 6 & -12 & -10 & -16 & -18 \\
\hline 7 & -14 & -12 & -25 & -30 \\
\hline 8 & -14 & -13 & -14.5 & -15 \\
\hline
\end{tabular}

when there is a failure of one element of the antenna Hence, for working diligently on the linear-array synthesis problem IPSOWM has been extensively used. As a matter of future scope, attempts are being made to improve upon the high parameters by implementing boundary variants of particle swarm optimization [15-17]. Correction in radiation pattern affected by mutual coupling is also being considered [18].

\section{References}

[1] Taskin, A., \& Gurel, C. S. (2003, October). Antenna array pattern optimisation in the case of array element failure. In 33rd European Microwave Conference Proceedings (IEEE Cat. No. 03EX723C)(Vol. 3, pp. 1083-1085). IEEE.

[2] Han, J. H., Lim, S. H., \& Myung, N. H. (2011). Array antenna TRM failure compensation using adaptively weighted beam pattern mask based on genetic algorithm. IEEE Antennas and Wireless Propagation Letters, 11, 18-21.

[3] Peters, T. J. (1991). A conjugate gradient-based algorithm to minimize the 
sidelobe level of planar arrays with element failures. IEEE Transactions on Antennas and Propagation, 39(10), 1497-1504.

[4] Wang, L. L., \& Fang, D. G. (2003, October). Combination of genetic algorithm and fast Fourier transform for array failure correction. In $6^{\text {th }}$ International SYmposium on Antennas, Propagation and EM Theory, 2003. Proceedings. 2003 (pp. 234-237). IEEE.

[5] Lozano, M. V., Rodríguez, J. A., \& Ares, F. (1999). Recalculating linear array antennas to compensate for failed elements while maintaining fixed nulls. Journal of electromagnetic waves and applications, 13(3), 397-412.

[6] Yeo, B. K., \& Lu, Y. (2005, December). Adaptive array digital beamforming using complex-coded particle swarm optimization-genetic algorithm. In 2005 AsiaPacific Microwave Conference Proceedings (Vol. 2, pp. 3-pp). IEEE.

[7] Balanis, C. A. (2016). Antenna theory: analysis and design. John wiley \& sons.

[8] Rodriguez, J. A., Ares, F., Moreno, E., \& Franceschetti, G. (2000). Genetic algorithm procedure for linear array failure correction. Electronics Letters, 36(3), 196198.

[9] Panigrahi, T., Patnaik, A., Sinha, S. N., \& Christodoulou, C. G. (2008, July). Amplitude only compensation for failed antenna array using particle swarm optimization. In 2008 IEEE Antennas and Propagation Society International Symposium (pp. 1-4). IEEE.

[10] Mandal, D., Ghoshal, S. P., \& Bhattacharjee, A. K. (2013). Optimized radii and excitations with concentric circular antenna array for maximum sidelobe level reduction using wavelet mutation based particle swarm optimization techniques. Telecommunication Systems, 52(4), 2015-2025.

[11] Mandal, D., Bhattacharjee, A. K., \& Ghoshal, S. P. (2011, February). Linear antenna array synthesis using improved Particle Swarm Optimization. In 2011 Second International Conference on Emerging Applications of Information Technology (pp. 365-368). IEEE.

[12] Mandal, D., Sadhu, D., \& Ghoshal, S. P. (2011). Thinned concentric circular array antennas synthesis using improved particle swarm optimization. ACEEE International Journal on Communication (IJCom), 2(2), 21-25.

[13] Mangoud, M. A. A., \& Elragal, H. M. (2009). Antenna array pattern synthesis and wide null control using enhanced particle swarm optimization. Progress in Electromagnetics Research, 17, 1-14.

[14] Bera, R., Lanjewar, R., Mandal, D., Kar, R., \& Ghoshal, S. P. (2015). Comparative study of circular and hexagonal antenna array synthesis using improved particle swarm optimization. Procedia Computer Science, 45, 651-660.

[15] Chakravorty, P., \& Mandal, D. (2015, December). Role of boundary dynamics in improving efficiency of particle swarm optimization on antenna problems. In 2015 IEEE Symposium Series on Computational Intelligence (pp. 1157-1163). IEEE.

[16] Chakravorty, P., \& Mandal, D. (2015). Hysteretic boundary conditions for PSO of antenna array pattern synthesis. Procedia Computer Science, 45, 628-634.

[17] Chakravorty, P., \& Mandal, D. (2015). PSO in concentric circular arrays for side lobe reduction with symmetric relocation boundary condition. In Intelligent 
Computing and Applications (pp. 503-509). Springer, New Delhi.

[18] Chakravorty, P., \& Mandal, D. (2016). Radiation pattern correction in mutually coupled antenna arrays using parametric assimilation technique. IEEE Transactions on Antennas and Propagation, 64(9), 4092-4095.

Submitted 28.02.2019

Accepted 03.05.2019 